\title{
Management of unscheduled tetanus prophylaxis in Emergency Departments: Point-of-Care implementation as a rapid tool for the evaluation of anti-tetanus antibodies
}

\author{
Andrea Rocchetti, ${ }^{1}$ Rossana Uberti, ${ }^{2}$ Carolina Prevaldi, ${ }^{3}$ Clara Maria Lazzara, ${ }^{1}$ Federica Riccio ${ }^{4}$ \\ 1 Microbiology Unit, Department of Hospital Services, SS. Antonio e Biagio e C. Arrigo Hospital, \\ Alessandria; ${ }^{2}$ Department of Surgery and Emergency Care, SS. Antonio e Biagio e C. Arrigo \\ Hospital, Alessandria; ${ }^{3}$ Department of Surgery and Emergency Care, Veneto Orientale Local Health \\ Unit 10, Portogruaro; ${ }^{4}$ Department of Public Health, Hygiene Section, University of Pavia, Italy
}

\section{Summary}

Background and aim: Each analytical activity, including those carried out in Point of Care (POCT) must be, at law, under the control of Laboratory Medicine. Before the implementation of the rapid tetanus quick stick (TQS) test for the evaluation of the specific tetanus immunisation, a multi-disciplinary and multi-professional group was created. The aim of this study was to evaluate the ability of Emergency Department (ED) staff to manage and correctly understand the result of TQS test in POCT.

Materials and Methods: This analysis took into consideration 152 patients admitted to ED with traumatic wounds; information on the state of tetanus immunisation at their arrival wasn't recorded. Blood sample analysis was performed twice. The Laboratory confirmed a $100 \%$ concordance between their results and ELISA test (standard criterion). Study design consisted of 2 phases: the first one (50 test) to preliminarily evaluate if any corrective action or improvement of procedures is required, and the second one (102 tests) to confirm the quality of corrective actions.

Correspondence: Andrea Rocchetti, Presidio 0spedaliero SS Antonio e Biagio, Via Venezia 16, 15121 Alessandria, Italy.

Tel.: +39.0131 .206312 - Fax: +39.0131 .206854 .

E-mail: arocchetti@ospedale.al.it

Key words: Tetanus; point of care testing; tetanus quick stick; immunisation.

Contributions: the authors contributed equally.

Conflict of interest: the authors declare no potential conflict of interest.

Received for publication: 3 February 2015.

Revision received: 1 December 2015.

Accepted for publication: 9 December 2015.

(C) Copyright A. Rocchetti et al., 2016

Licensee PAGEPress, Italy

Microbiologia Medica 2016; 31:5082

doi:10.4081/mm.2016.5082

This article is distributed under the terms of the Creative Commons Attribution Noncommercial License (by-nc 4.0) which permits any noncommercial use, distribution, and reproduction in any medium, provided the original author(s) and source are credited.
Results: The concordance of results between TQS test in Laboratory and POCT test in ED was $80 \%$ in the first phase and $95 \%$ in the second one.

Conclusions: The use of the rapid TQS test is a valuable tool; however, to avoid serious mistakes of interpretation, periodic checks on the quality of the results must be arranged.

\section{Introduction}

Tetanus is an acute, often fatal, infection caused by Clostridium tetani, a gram positive, anaerobic and spore-forming bacillus $(1,8)$.

It is well known that tetanus is sporadic in several industrialised countries; the availability of highly-safe and effective childhood primary vaccination programs has made a major contribution to the drastic reduction of morbidity and deaths, although an increasing number of cases still occur among the elderly (7).

Tetanus remains significantly prevalent in developing countries, where mortality is high particularly among the neonates (9).

In 2010 (the last year for which data are available), in Europe, case rate was as low as 0.02 per 100.000 population, while the Italian case rate was approximately 0.09 per 100.000 . Italy has been continuously reporting the highest member of tetanus cases since 2006, ranging between 53-64 cases per year (2).

C. tetani is a common soil bacterium, its spores are ubiquitous in nature and are found in the soil and in the intestines and faeces of animals and humans. The production of spores allows the microorganism to remain in hostile environments (soil, metal) for years.

When the spore is transformed into the vegetative form, it is able to produce two toxins: the tetanolysin with haemolytic activity and the tetanospasmin (or tetanus toxin), a thermolabile protein highly virulent, neurotoxic, causing the clinical symptoms of the disease.

Tetanospasmin penetrates in the axons and goes back to the neurons of the spinal cord and brain stem, reaching the terminations of the movement secreting inhibitory glycine and GABA and causing the destruction of the apparatus protein, responsible for the release of these two neurotransmitters. The clinical outcome of this process is the spastic paralysis caused by lack of motor inhibition, with simultaneous contraction of agonist and antagonist muscles. The results can be fatal when the paralysis affects the respiratory muscles.

The incubation period for tetanus varies from 3 to 21 days. Generally, the shorter the incubation period is, the more severe the clinical course is. 
The most common symptoms include fever, headache, irritability and pain at the site of inoculation. The beginning of the period of state (period of clinical manifestations) is identified by the appearance of tonic contractions. Generalized tetanus is the most common form (compared to chefalic, localized and neonatal tetanus) and is seen in $80 \%$ of cases. Trismus is often the presenting symptom, caused by severe contraction of the masseter, leading to the lay term lockjaw. Classic advanced signs of tetanospasm are opisthotonus (severe contraction of muscle in hyperextention) and risus sardonicus, literally the sardonic smile (2). The diagnosis of tetanus is made on a clinical basis.

The spores can penetrate the human body through penetrating wounds (barbed-wire) or grazes (falls, glass, stones, agricultural tools). Further attention should be given to scratches caused by rose thorns.

The traumatic wounds represent a significant proportion among the accesses to the Emergency Department (ED, about 5.4\%). In 10.3\% of all patients, procedures regarding wound care occur.

In case of traumatic injury, before proceeding to tetanus prophylaxis, the immunization status of patients should be checked through the consultation of clinical documentation confirming vaccination/recall of vaccination (usually available in $1 \%$ of cases). In absence of reliable data we resort to a medical history (10).

The tetanus vaccination status of a patient is often difficult to be established, especially when the patient is unable to remember if there has been a vaccination/recall of vaccination or mistake tetanus vaccination with other types of vaccination or different intravenous therapies. The patient may also be unconscious, intoxicated or with cognitive deficits, cannot understand Italian or may have never had a full vaccination cycle. In these cases, it is helpful to use a quick test to assess the immune status of the patient (3).

The World Health Organization acknowledges the ELISA test as the standard criterion, with a threshold of $0.1 \mathrm{IU} / \mathrm{mL}$, to diagnose tetanus immunization status. This threshold is 10 times higher than the serum antitoxin level considered to be protective (5). It is a quantitative method that provides guidance on the need for basic immunization or a booster vaccine. ELISA is not readily available in ED.

TQS (Tetanus Quick Stick), instead, is a rapid test in immunochromatography, which has already been used for several years in Europe, especially in France; it shows in 10 minutes if the antibody level is above $0.1 \mathrm{IU} / \mathrm{mL}$ or not.

The TQS test allows to evaluate the immune status of patients with traumatic injury and to avoid under/over immunization in the absence of reliable information on their specific vaccination status.

The purpose of this paper is to describe some aspects considered critical in the implementation of the rapid test TQS at ED in the Hospital of Alexandria, Italy.

POCT (Point of Care Testing) means that a laboratory test is performed by health staff who do not have any formal qualification regarding such activities, through the use of easy-to-use instrumentation, usually in blood whole.

\section{Materials and Methods}

The Laboratory Medicine, in order to analyse all aspects of the management and plan correctly the interventions, has established a working group consisting of a doctor and a nurse of ED, a microbiologist and a laboratory technician.

The team has defined all organizational aspects related to the implementation of the rapid test TQS in POCT such as: analysis of scientific literature, workloads, staff training, supply management, quality control, clinical audit, tracking results, cost analysis. The analysis was carried out early in the year 2013 .
After the training of clinical operators (11 doctors and 30 nurses), the test TQS was introduced on an experimental basis in the ED, after verification of the analytical concordance of $100 \%$ with the gold standard ELISA test. The goal of our work was to evaluate the ability of staff to manage ED and interpret correctly the test result TQS in POCT, before its final implementation.

The analysis has taken into consideration 152 samples of patients referred in the ED with traumatic wounds; it was not possible to get information on their state of tetanus immunization. Sampling was performed in duplicate: the same sample was performed with the same test both in ED and in Laboratory.

The reagents used were the following.

Rapid test in immunochromatography: TQS tetanus quick stick (Nephrotek Laboratory, Rungis, France). The test is positive when the blood sample is an antibody titre of at least $0.1 \mathrm{IU} / \mathrm{mL}(6,11)$. The test is positive with titles $\geq 0.1 \mathrm{IU} / \mathrm{mL}$ of serum and $\geq 0.2 \mathrm{IU} / \mathrm{mL}$ of whole blood.

Immunoassay test: Serion ELISA Classic Tetanus IGG (Institut Virion/Serion GmbH, Würzburg, Germany). It's a quantitative test which allows to determine the immune status of the patient by providing directions for the use of immunoprophylaxis.

\section{Results}

In 2013, 152 samples from patients referred to ED with traumatic injury were evaluated; their status of immunization was unknown but the total number of samples was confirmed in ELISA with the concordance of $100 \%$ by Medicine Laboratory. It was chosen to analyse the first 50 samples, in order to evaluate any corrective action or improvement of procedures.

Out of 50 samples, 40 (80\%) were concordant between TQS performed in POCT and TQS in Laboratory. In 10 cases results are discordant because they were interpreted as negative in POCT and as positive in the Laboratory. Of these 8 samples tests showed a weak result in the test region (T-line) and the confirmation in ELISA showed antibody titles between 0.1 and $0.5 \mathrm{IU} / \mathrm{mL}$; in the other 2 cases the test region (Tline) was well defined, leaving no doubts as to interpretation on the positive test (Tables 1 and 2).

After returning the results of the study to the ED staff, it was decided to train again all nurses and doctors on the correct reading and interpretation of the test.

In the second phase were evaluated 102 samples and the concordance between POCT and Laboratory test get on from $80 \%$ to $95 \%$.

The discordant cases were 5: in 2 cases, the tests performed in ED TQS was interpreted as negative, while the same TQS test performed in the laboratory was taken as positive; tests showed a weak band in the test and confirmation in ELISA antibody titres showed between 0.1 and $0.5 \mathrm{IU} / \mathrm{mL}$ and in 3 cases the test run in TQS ED was interpreted as positive while the same TQS test performed in the laboratory was negative; tests did not show any band in the test and confirmation in ELISA did not show the presence of antibodies (Tables 1 and 2).

\section{Discussion}

If the immune status of patients presenting in ED with traumatic wounds is unknown, it is often impossible to avoid over/under immunization, therefore it is useful to perform a quick test in POCT able to discriminate patients with antibody title of at least $0.1 \mathrm{IU} / \mathrm{mL}$, title considered protective.

The TQS test performed in the laboratory shows a 100\% concordance 
Table 1. Comparison between Tetanus Quick Stick (TQS) test in Emergency Department (ED) and Tetanus Quick Stick test in Laboratory before and after training.

\begin{tabular}{|c|c|c|c|c|c|}
\hline Sample & $\begin{array}{l}\text { TQS } \\
\text { (ED) }\end{array}$ & $\begin{array}{l}\text { Discordant } \\
\text { sample }\end{array}$ & $\begin{array}{c}\text { ELISA } \\
\text { (Laboratory) UI/mL }\end{array}$ & $\begin{array}{c}\text { TQS } \\
\text { (Laboratory) }\end{array}$ & $\begin{array}{l}\text { Discordant } \\
\text { sample }\end{array}$ \\
\hline \multicolumn{6}{|c|}{ Before training } \\
\hline Positive & 31 & 0 & 41 & 41 & 0 \\
\hline Negative & 19 & 10 & 9 & 9 & 0 \\
\hline Total & 50 & 10 & 50 & 50 & 0 \\
\hline \multicolumn{6}{|c|}{ After training } \\
\hline Positive & 69 & 2 & 70 & 70 & 0 \\
\hline Negative & 33 & 3 & 32 & 32 & 0 \\
\hline Total & 102 & 5 & 102 & 102 & 0 \\
\hline
\end{tabular}

Table 2. Comparison between Tetanus Quick Stick (TQS) test and ELISA test in Laboratory before and after training.

\begin{tabular}{lccc} 
Sample & $\begin{array}{c}\text { TOS } \\
\text { (ED) }\end{array}$ & $\begin{array}{c}\text { ELISA } \\
\text { (Laboratory) }\end{array}$ & $\begin{array}{c}\text { TOS } / \mathrm{mL} \\
\text { (Laboratory) }\end{array}$ \\
\hline 1 & Before training & \\
2 & Negative & 4.85 & Positive \\
\hline 3 & Negative & 0.4 & Positive \\
\hline 4 & Negative & 0.21 & Positive \\
\hline 5 & Negative & 0.27 & Weak positive \\
\hline 6 & Negative & 0.51 & Positive \\
\hline 7 & Negative & 0.22 & Weak positive \\
\hline 8 & Negative & 0.21 & Weak positive \\
10 & Negative & $>500$ & Positive \\
\hline \hline & Negative & 0.42 & Positive \\
\hline 1 & Negative & 0.58 & Positive \\
\hline 2 & & After training & \\
\hline 3 & Positive & 0.072 & Negative \\
4 & Positive & 0 & Negative \\
\hline 5 & Negative & 0.97 & Positive \\
\hline & Negative & 0.2 & Weak \\
\hline & Negative & 0.73 & Positive \\
\hline
\end{tabular}

with the gold standard in the ELISA test and it is therefore reliable.

The management of the test TQS in ED presents difficulties related to the interpretation of the analytical result probably related to the organizational complexity of the setting operating (overcrowding, high turnover of staff etc).

The percentage of agreement between the results increase in the second phase of the study, after the involvement of all the staff of ED, underlining the need for constant feedback between the Laboratory and the ED. It is clear that the responsibility for all activities of Laboratory Medicine included POCT can be attributed to the Director of the Laboratory, who can use subordinate operators, even functionally, to obtain a periodic check of the correct execution of all the rapid tests introduced in POCT, although simple to perform and interpretation, in order to avoid serious mistakes.

\section{Conclusions}

The use of a rapid diagnostic Point of Care test for the evaluation of patients' tetanus immunisation state, is a valuable tool to avoid over/under immunisation against tetanus toxin; however, it is necessary to program periodic checks on the quality of the results (4).

\section{References}

1. Adams EB, Laurence DR, Smith JWG. Tetanus. Oxford: Blackwell Scientific; 1969.

2. Annual epidemiological report on communicable diseases in Europe 2012. Tetanus. Surveillance Report. European Center for disease prevention and control.

3. Colombet I, Saguez C, Coudert B, et al. Diagnosis of tetanus immunization status: multicenter assessment of a rapid biological test. Clin Diagn Lab Immunol 2005;12:1057-62.

4. Elkharrat D, Boyer-Chammard A, Raskine L, et al. Impact of guidelines to alter antitetanus prophylaxis practices and reduce costs in the emergency department. Am J Ther 1999;6:203-9.

5. Gazalka A. The immunological basis for immunization series. Module 3: tetanus. global programme for vaccines and immunization, expanded programme on immunization. Geneva: World Health Organization; 1993.

6. Hatamabadi HR, Abdalvand A, Safari S, et al. Tetanus Quick Stick as an applicable and cost-effective test in assessment of immunity status. Am J Emerg Med 2011;29:717-20.

7. Quinn HE, McIntyre PB. Tetanus in elderly: an important preventable disease in Australia. Vaccine 2007;25:1304-9.

8. Rhee P, Nunley M, Velmahos G, et al. Tetanus and trauma: a review and recommendations. J Trauma 2005;58:1082-8.

9. Roper MH, Vandelaer JH, Gasse FL. Maternal and neonatal tetanus. Lancet 2007;370:1947-59.

10. Talan DA, Abrahamian FM, Moran GJ, et al. Tetanus immunity and physician compliance with tetanus prophylaxis practices among emergency department patients presenting with wounds. Ann Emerg Med 2004;43:305-14.

11. Touzè $M$, Potel $\mathrm{G}$, Le Kieffre $\mathrm{P}$, et al. Immunité anti-tétanique desblessés admis dans le services urgences. Reanim. Urgences 1992;1:910-4. 